\title{
Lagrangian Density and Local Symmetries of Inhomogeneous Hyperconical Universes
}

\author{
Robert Monjo \\ Dpto de Álgebra, Geometría y Topología, Fac. CC. Matemáticas, Universidad \\ Complutense de Madrid, Plaza de Ciencias 3, E-28040 Madrid, Spain \\ Tel.: +34 913947609 \\ rmonjo@ucm.es
}

\section{Rutwig Campoamor-Stursberg}

Dpto de Álgebra, Geometría y Topología, Fac. CC. Matemáticas, Universidad Complutense de Madrid, Plaza de Ciencias 3, E-28040 Madrid, Spain rutwig@ucm.es 
June 2020

\begin{abstract}
Hyperconical universes can be represented by means of an inhomogeneous metric with positive curvature and linear expansion, that is isomorph to flat universes with acceleration thanks to an appropriate transformation. Various symmetry properties of this metric are analysed, primarily at the local scale. In particular, the Lagrangian formalism and the Arnowitt-Deser-Misner (ADM) equations are applied. To this extent, a modified Gravity Lagrangian density is derived, from which the comoving paths as solutions of the Euler-Lagrange equations leading to a stationary linear expansion are deduced. It is shown that the evolution of this alternate metric is compatible with the ADM formalism when applied to the modified Lagrangian density, thanks to a redefinition of the energy density baseline (according to the global curvature). Finally, results on symmetry properties provide that only the angular momenta are global symmetries. The radial inhomogeneity of the metric is interpreted as an apparent radial acceleration, which breaks all the non-rotational local symmetries at large distances.
\end{abstract}

Keywords: Modified Lagrangian density; Hyperconical Universe; Symmetry. PACS numbers: 04.20Fy, 04.90e

\title{
1. Introduction
}

\subsection{Motivation}

The standard $\Lambda$ CDM theory is based on the assumption that General Relativity (GR) and the Einstein field equations are valid for the Universe as a whole. Moreover, hypothesis of homogeneity and isotropy are taken to choose the family of possible metrics for the Universe [1]. Modified $f(R)$ gravities and $f(T, R)$ extended teleparallel models attempt to generalise the frame by assuming that local limit approaches to the GR $[2,3,4,5]$. Moreover, if the homogeneity hypothesis is not imposed, it is possible to explain the accelerated Universe's expansion by using alternatives such as the timescape cosmology of Wiltshire and others, or models based on back-reaction effects of cosmic inhomogeneities $[6,7,8]$.

At the local limit, the $\Lambda \mathrm{CDM}$ theory uses the Friedmann-Lemaitre-RobertsonWalker (FRW) universes, which consider that space and temporal factors are separable [9]. Let $\vec{\ell}$ be spatial coordinates of the Universe; so they can be rewritten by using comoving coordinates $\overrightarrow{\ell^{\prime}}$ and a scaling factor $a(t)$ varying with time $t$. For instance, if we choose a Universe characterised by $S_{R}^{3}:=\left\{\vec{\ell} \in \mathbb{R}^{4}:|\vec{\ell}|=R(t)\right\}$, i.e. an expanding 3 -sphere of radius $R(t) \in \mathbb{R}_{\geq 0}$ and centred in the origin (singularity in $t=0$ ), the scaling factor is $a(t)=R(t) / R\left(t_{o}\right)$ and total spatial coordinates are $\vec{\ell}=a(t) \overrightarrow{\ell^{\prime}} \in S_{R}^{3}$. Therefore, the line element contains both spatial and temporal differentials, i.e., $d \ell=$ $a(t) d \ell^{\prime}+\ell^{\prime} d a(t)$. If the second term is absorbed, a radial inhomogeneity appears in the spatial term. It follows that the FRW model does not include the simplest expanding 3 -sphere according to $R(t)=t$, embedded in $\mathbb{R}^{5}$. If the Hubble parameter is defined as $H:=\dot{a} / a$, this linear expansion corresponds to $H=1 / t$, which is compatible with the 
empirical age of the universe $[10,13,11,12]$. For this reason, alternative cosmological models are based on the equality $1 / H=t$, such as the Dirac-Milne model and the inhomogeneous hyperconical model $[14,15,16,17]$. However, symmetry properties of these universes have not been analysed yet, so it is necessary to explore conserved quantities and contrast them with physical observables.

The aim of this paper is to analyse the symmetry properties of a radially inhomogeneous hyperconical model according to the Killing vectors of the metric, the Lagrangian formalism and the Arnowitt-Deser-Misner (ADM) equations [18, 19, 20]. This work expands new results obtained from the family of metrics proposed in [16, 17]. The model is also based on the same hypothesis of linear expansion as [14, 15], but analyses the curvature tensor according to the point of view of a hypothetical observer located in the hypersurface of an expanding universe (hypercone section), which can have both positive or negative curvature. This contrasts with the Dirac-Milne universe, which assumes a zero or negative curvature in a classic FRW metric [14].

Assuming hyperconical universes with linear expansion, isotropic but radially inhomogeneous metrics are obtained by transformations that preserves the proper time. These metrics are compatible with observations of luminosity distance of $580 \mathrm{SNe}$ Ia from the Supernova Cosmology Project [16]. Moreover, there exists a family of locally conformal transformations that lead the (linearly expanding and curved) metrics to become the flat FRW metric, absorbing the spatial inhomogeneity as an acceleration in time. That predicted a dark energy density about $\Omega_{\Lambda}=0.6937181(2)$ (presented in [17]), compatible with the standard-model-dependent observation $\left(\Omega_{\Lambda}=0.690(6)\right)$ $[21,22]$. The relation between radial inhomogeneity, Lagrangian curvature terms and effective dark energy is also found in other modified gravities, but without a prediction of its value $[23,24,25,26,7,27]$.

In order to present new results, this work requires some previous sections. Specifically to remind notions of local symmetries under Lagrangian formalism, the paper firstly revisits generalities on Field Theory and the ADM formalism. The second section is a summary to describe the Inhomogeneous Hyperconical Universe model, and the following sections show results on the modified Lagrangian density for that universe and the local symmetry properties. Finally, main conclusions close the paper.

\subsection{Generalities on Field Theory}

To fix the notations used in this paper, it is required a brief reminder of generalities on the classical Field Theory. Let $M$ be a spacetime manifold with the target space $\mathcal{C}$ determined by the values of the fields at arbitrary points. Taking $m$ real-valued scalar fields, $\phi_{1}, \ldots, \phi_{m}$, the target manifold is $\mathbb{R}^{m}$. We assume $\mathcal{S}[\phi]$ is the integral over $M$ of a function called Lagrangian density $\mathcal{L}$, which depends on $\phi$, its derivative and the position, $\mathcal{L}\left(\phi, \partial_{\mu} \phi, x^{\mu}\right)$. Note that the classical Lagrangian $L$ is the volume integral of the Lagrangian density $\mathcal{L}$, i.e. $L=\int d^{n} x \mathcal{L}$. The action functional $\mathcal{S}: \mathcal{C} \rightarrow \mathbb{R}$, is given 
Lagrangian density and symmetries of inhomogeneous hyperconical universes ${ }^{A} T_{E} X{ }_{\varepsilon} 4$

by:

$$
\mathcal{S}[\phi]=\int_{M} \mathcal{L}\left[\phi(x), \partial_{\mu} \phi(x), x\right] \mathrm{d}^{n} x
$$

In addition to this, some boundary conditions are required The Euler-Lagrange equations are:

$$
\partial_{\mu}\left(\frac{\partial \mathcal{L}}{\partial\left(\partial_{\mu} \phi\right)}\right)-\frac{\partial \mathcal{L}}{\partial \phi}=0
$$

Definition. A solution subspace of $\mathcal{C}$ is called an on-shell solution if it consists of functions $\phi$ such that all functional derivatives of $\mathcal{S}$ at $\phi$ are zero, that is:

$$
\frac{\delta \mathcal{S}[\phi]}{\delta \phi(x)} \approx 0
$$

and that $\phi$ satisfies the given boundary conditions. If a functional derivative $D$ can be lead to off shell, we say $D$ generates an off-shell symmetry. If this only holds on shell, we say $D$ generates an on-shell symmetry. Then, we say $D$ is a generator of an one-parameter symmetry Lie group [28].

Theorem. (Noether current) Given an infinitesimal transformation on $\mathcal{C}$, generated by a functional derivation $D$ such that

$$
D\left[\int_{M} \mathcal{L} \mathrm{d}^{n} x\right]=\int_{\partial M} F^{\mu}[\phi(x), \partial \phi, \partial \partial \phi, \ldots] \mathrm{d} s_{\mu}
$$

for all compact manifolds $M$, i.e. $D[\mathcal{L}(x)] \approx \partial_{\mu} F^{\mu}(x)$ for all $x$, where we set $\mathcal{L}(x)=\mathcal{L}\left[\phi(x), \partial_{\mu} \phi(x), x\right]$. Then the following quantity:

$$
J^{\mu}:=\frac{\partial \mathcal{L}}{\partial\left(\partial_{\mu} \phi\right)} D[\phi]-F^{\mu}
$$

satisfies the continuity equation $\partial_{\mu} J^{\mu}=0$.

Therefore, $J^{\mu}$ is the Noether current associated with the symmetry. If we consider noncompact manifolds $M$ with currents fall off sufficiently fast at infinity, the current can be integrated over a space-like slice $\mathcal{X}$. In this case, the continuity equation implies that we get a conserved quantity $\mathcal{Q}$ called the Noether charge:

$$
\mathcal{Q}:=\int_{\mathcal{X}} J^{0} d^{3} x
$$

\subsection{ADM Formulation of Gravity}

In this work we denote by $\mathbb{R}_{\eta}^{1, n}:=\left(\mathbb{R}^{n+1}, \eta_{1, n}\right)$ the flat Lorentzian manifold, where the metric tensor is given by $\eta_{1, n}:=\operatorname{diag}(1,-1, \ldots,-1)$. Taking $n=3$ and normalising the gravitational constant $16 \pi \mathrm{G} \equiv 1$, the general action should be:

$$
\mathcal{S}=\int d^{4} x \sqrt{-g}\left(f(R)+\mathcal{L}_{M}\right) \approx \int d^{4} x \sqrt{-g}\left(R-\Lambda+\mathcal{L}_{M}\right)
$$

where $R$ is the Ricci scalar curvature, $\Lambda$ is the cosmological constant and $\mathcal{L}_{M}$ is the mass term. In fact, $f(R)$ can be the standard Lagrangian density $(R-\Lambda)$ from the EinsteinHilbert action, or can be any function of modified gravity depending on scalars built using 
the Riemann curvature tensor $R_{\alpha \beta \gamma}^{\rho}$ or the Ricci curvature $R:=g^{\alpha \gamma} R_{\alpha \beta \gamma}^{\beta}$ among others, or using torsion tensor $f(T)$ like in parallel gravities $[29,5]$. With $G_{\alpha \beta}:=R_{\alpha \beta}-g_{\alpha \beta} R / 2$ being the Einstein tensor, the field equations are given by:

$$
G_{\alpha \beta}-\Lambda g_{\alpha \beta}=8 \pi \mathrm{G} T_{\alpha \beta},
$$

where $T_{\alpha \beta}:=-2 \delta \mathcal{L}_{M} / \delta g^{\alpha \beta}+g_{\alpha \beta} \mathcal{L}_{M}$ is the stress-energy tensor. If the space-time of the universe is foliated into a family of space-like surfaces, we can introduce a Hamiltonian formulation according to the Arnowitt-Deser-Misner (ADM) formalism [18, 19]. This is useful because, from the Hamiltonian $\mathcal{H}:=p \dot{q}-\mathcal{L}$, a set of equations of motion can be obtained for the generalized coordinates $q$ and momenta $p$ as $\dot{q}=\partial \mathcal{H} / \partial p$ and $\dot{p}=-\partial \mathcal{H} / \partial q$. Still according to the ADM formalism, the spatial submanifold can be interpreted as an embedding into the global space-time and thus the metric elements can be separated in a temporal part $g_{00}$ and another spatial part $g_{i j}$. Moreover, ADM takes $\mathcal{L}_{M}=0=\Lambda$ and then Lagrangian density can be rewitten as:

$$
\mathcal{L}=-g_{i j} \partial_{t} \pi^{i j}+l h+2 l_{i} \pi^{i j}{ }_{; j}-2 \partial_{i} s^{i}
$$

where $l:=\left(g^{00}\right)^{-1 / 2}$ is the lapse, $l_{i}:=g_{0 i}$ is the shift, $\pi^{i j}$ are conjugate momenta, $h$ is the Hamiltonian constraint and $s^{i}$ are auxiliary momenta:

$$
\begin{aligned}
\pi^{i j} & =\sqrt{-g}\left(\Gamma_{p q}^{0}-g_{p q} \Gamma_{r s}^{0} g^{r s}\right) g^{i p} g^{j q} \\
h & =-\sqrt{g_{s}} R_{k}^{k}-\frac{1}{\sqrt{g_{s}}}\left(\frac{1}{2} \pi^{2}-\pi^{i j} \pi_{i j}\right) \\
s^{i} & =l_{0 j} \pi^{i j}-\frac{1}{2} l^{0 i} \pi+\nabla^{i} \sqrt{-g}
\end{aligned}
$$

where $l^{i}:=g^{0 i} / g^{00}, \pi_{i j}:=g_{i k} g_{j l} \pi^{k l}, \pi=g_{i j} \pi^{i j}, g:=\operatorname{det} g_{\mu \nu}$ is the determinant of the metric and $g_{s}:=\operatorname{det} g_{i j}$ is the determinant of the spatial metric. The ADM equations for the metric evolution are:

$$
\partial_{t} g_{i j}=2 \frac{1}{\sqrt{-g_{s} g^{00}}}\left(\pi_{i j}-\frac{1}{2} \pi g_{i j}\right)+\nabla_{i} g_{0 j}+\nabla_{j} g_{0 i}
$$

In this context, it is of special interest to study the evolution of the metrics with nonzero lapse $\left(g_{00} \neq 1\right)$ and shift $\left(g_{0 r} \neq 1\right)$. A particular case is given by an inhomogeneous universe built from an appropriate transformation of the hyperconical metric (Sec. 2).

\section{Inhomogeneous Hyperconical Universe}

\subsection{Hypothesis}

A summary of the used model is described in this section, according to [16, 17]. Let $M \subset \mathbb{R}_{\eta}^{1,4}$ be a support manifold contained in the 5-dimensional Minkowski space, more specifically $M:=\left(\mathbb{R}_{\geq 0} \times \mathbb{R}^{4}, \eta_{1,4}\right)$, with inner product $(\cdot)$ given by the usual Lorentzian metric $\eta$ of signature $(1,4)$. In order to restrict the 5-dimensional support manifold $M$ to the 4-dimensional $\mathrm{H}^{4}$, we take into account a constraint based on a hyperconical 
universe with linear expansion. More specifically, we focus on an expanding distance between two points $X, O \in M$ as a function of an observable time $t \in \mathbb{R}_{\geq 0}$ :

$$
\mathrm{H}^{4}:=\left\{X \in M:|X-O|_{\eta_{1,4}}=\beta_{o} t\right\} .
$$

Here $\beta_{o} \in \mathbb{C}$ is assumed as a constant with respect to the time $t \in \mathbb{R}_{\geq 0}$ but dependent on the chosen origin $O \in M$.

Let $C:=\left(T_{O} M, I_{d}, \eta_{1,4}\right)$ be a coordinate system or chart such that the coordinates of the points $X, O \in T_{O} M$ are respectively $X=\left(x^{0}, \ldots, x^{4}\right)$ and $O=(0, \ldots, 0)$, with the identity $I_{d}$ mapping. However, the coordinates of $X$ are rewritten as $\left(t_{X}, \vec{r}, u\right)$ for convenience, where $\vec{r}:=\left(x^{1}, x^{2}, x^{3}\right) \in \mathbb{R}^{3}$ is the ordinary 3 -vector, $u:=x^{4} \in \mathbb{R}$ is the additional spatial dimension and $t_{X}:=x^{0} \in \mathbb{R}_{\geq 0}$ is the time dimension. Choosing $t=t_{X}$, the condition of the hypersurface $\mathrm{H}^{4}$ is now $\nu^{2} t^{2}-\vec{r}^{2}-u^{2}=0$ with $\nu^{2}:=1-\beta_{o}^{2}$. Note that the hypercone with $\nu>0$ is an asymptotic limit of hyperboloid manifolds. In fact, if the constraint is taken as $t^{2}-\vec{r}^{2}-u^{2}=\alpha$ with constant $\alpha \neq 0$, the spaces are known as de Sitter universes [30, 31]. Moreover, $\mathrm{H}^{4}$ is not a Dirac-Milne universe because it admits positive spatial curvature.

Remark. The manifold $\left(\mathrm{H}^{4}, \eta_{1,4}\right)$ is spatially homogeneous. The homogeneity of $\mathrm{H}^{4}$ is verifiable because it can be foliated by spatial hypersurfaces $\Sigma_{t}$ such that, for $\forall p, q \in \Sigma_{t}$ and $\forall t$, there exists a transformation (diffeomorphism) carrying the point $p$ to point $q$ and leaving the metric invariant. In other words, a spheroidal submanifold $S_{t}^{3}$ can be defined for each time $t$ as the intersection between the hypercone $\mathrm{H}^{4}$ and the isochronous hyperplane at this time $t$ :

$$
S_{t}^{3}:=\left\{(\vec{r}, u) \in \mathbb{R}^{4}: \vec{r}^{2}+u^{2}=\nu^{2} t^{2}\right\} \subset \mathrm{H}^{4}
$$

That is, the time coordinate $t$ is considered as the age of the universe (radius) and the $t$-isochronous 3 -spheroid $S_{t}^{3}$ could be homeomorph to our expanding spatial universe (Eddington idea), locally conformally flat. Consequently, the universe manifold is globally hyperbolic, i.e, each $S_{t}^{3}$ is also a Cauchy surface $\ddagger$ [32].

Remark. The manifold $\left(\mathrm{H}^{4}, \eta_{1,4}\right)$ is spatially isotropic. Note that $\mathrm{H}^{4}$ can be covered by a set of timelike curves $\left\{\left.X\right|_{\gamma}\right\} \subset \mathrm{H}^{4}$ and $\left.\forall p \in X\right|_{\gamma}$ and $\forall v, w \in T_{p} S_{t}^{3}$ orthogonal to $\left.X\right|_{\gamma}$ there exists a transformation (diffeomorphism) leaving fixed $p$ and carrying $v$ to $w$, and leaving the metric invariant. These curves $\left\{\left.X\right|_{\gamma}\right\}$ are called comoving observers.

The expansion of the universe is an absolute movement with respect to the point $O=(0, \overrightarrow{0}, 0)_{C}$, which is fixed (respect to chart $C$ ). Therefore, the minimum movement of "particles" corresponds to the comoving observers with coordinates $(t, \overrightarrow{0}, \nu t)_{C} \subset \mathrm{H}^{4}$. These particles are interpreted as paths at rest with respect to the expanding universe $S_{t}^{3}$

$\ddagger$ A Cauchy surface is any subset of space-time which is intersected by every causal (timelike) curve exactly once. 
Lagrangian density and symmetries of inhomogeneous hyperconical universes ${ }^{A} T_{E} X{ }_{2} 7$

\subsection{Moving charts}

2.2.1. Problem on measuring proper time According to an expected equivalence, an observer that lives in $\mathrm{H}^{4}$ will measure local distances as in the Minkowskian space $\mathbb{R}_{\eta}^{1,3}:=\left(\mathbb{R}^{4}, \eta_{1,3}\right)$. Therefore, the proper time must be the same. For instance, let $x_{0}, x \in \mathbb{R}_{\eta}^{1,3}$ be two static points of an observer with coordinates $x_{0}=\left(t_{0}, \overrightarrow{0}\right)$ and $x=(t, \overrightarrow{0})$ with $0<t_{0}<t$. Their extended points in $\mathrm{H}^{4}$ are $x_{0}{ }^{\prime}=\left(t_{0}, \overrightarrow{0}, \nu t_{0}\right)$ and $x^{\prime}=(t, \overrightarrow{0}, \nu t)$, as we can take spaces $\mathbb{R}_{\eta}^{1,3}$ that intersect to $\mathrm{H}^{4}$ at points $x_{0}$ and $x$. We infer that there exists a smooth map $f:\left(\mathrm{H}^{4} \backslash\{O\}, \eta_{1,4}\right) \rightarrow\left(\mathbb{R}_{>0} \times \mathbb{R}^{3}, g\right):=\mathbb{R}_{g}^{1,3}$ such that the metric $g$ inherits the form of $\mathrm{H}^{4}$ but produces the same local $(\vec{r}=\overrightarrow{0})$ distance at rest in $\mathbb{R}_{g}^{1,3}$ as does for $\mathbb{R}_{\eta}^{1,3}$. As $x$ and $x_{0}$ are at rest for definition in $\mathbb{R}_{\eta}^{1,3}$, the distance turns out to be the proper time of the observer:

$$
t-t_{0}=\left|x-x_{0}\right|_{\eta_{1,3}}=\left|x-x_{0}\right|_{g}=\left|f\left(x^{\prime}\right)-f\left(x_{0}^{\prime}\right)\right|_{g}
$$

2.2.2. Physical solution Let $y^{\prime}(t)=(t, \vec{r}, \nu t), x^{\prime}(t)=(t, \overrightarrow{0}, \nu t) \subset\left(\mathrm{H}^{4}, \eta_{1,4}\right)$ be two comoving paths, considering the second one as an observer, i.e. taking $y_{0}^{\prime}:=y^{\prime}\left(t_{0}\right)$ and $x_{0}^{\prime}:=x^{\prime}\left(t_{0}\right)$ as a point of the past. If the observer performs measurements in $t_{0}$ and $t$, there exists a deformed path $y^{\prime \prime}(t):=\mathcal{T}_{t} y^{\prime}(t)$ intersecting in $\mathrm{H}^{4}$ during these measurements, with changes in $0<t_{0}<t$ due to a Deformation Operator, $\mathcal{T}_{t}$, such as

$$
\begin{aligned}
& \mathcal{T}_{t}:\left(\mathrm{H}^{4}, \eta_{1,4}\right) \rightarrow\left(M, \eta_{1,4}\right) \subset \mathbb{R}_{\eta}^{1,4} \\
& y_{0}{ }^{\prime}=\left(t_{0}, s\left(t_{0}\right)\right) \mapsto y_{0}{ }^{\prime \prime}(t):=\left(t_{0}, \frac{t}{t_{0}} s\left(t_{0}\right)\right) \subset M
\end{aligned}
$$

where $t_{0} \in \mathbb{R}_{>0}$ and $s(t):=(\vec{r}(t), \nu t) \subset \mathbb{R}^{4}$ are respectively the temporal and spatial components of the path $y(t)=(t, s(t))=(t, \vec{r}, \nu t) \subset \mathrm{H}^{4}$.

With this, $x^{\prime \prime}=x^{\prime}$ and $x_{0}{ }^{\prime \prime}=\left(t_{0}, \overrightarrow{0}, \nu t\right) \in \mathbb{R}^{1,4}$. Now, the $u$-component of the difference $x^{\prime}-x_{0}{ }^{\prime \prime}$ is zero, and the proper time is the same that Eq.(16). Therefore the metric $g$ of $\mathrm{H}^{4}$ is induced by the differential line of $x^{\prime}-x_{0}{ }^{\prime \prime}$ in $\mathbb{R}_{\eta}^{1,4}$ :

$$
\left.\mid d\left(x-x_{0}\right)\right)\left.\right|_{g} ^{2}=\left|d\left(f\left(x^{\prime}\right)-f\left(x_{0}{ }^{\prime}\right)\right)\right|_{g}^{2}=\left|d\left(x^{\prime}-x_{0}{ }^{\prime \prime}\right)\right|_{\eta_{1,4}}^{2}
$$

where the morphism $f:(t, \vec{r}, u) \mapsto(\hat{t}, \vec{r}) \in \mathbb{R}_{g}^{1,3}$ can be chosen such that $\hat{t}(\vec{r}, t)=t$, $\overrightarrow{\hat{r}}(\vec{r}, t)=\vec{r}, \forall \vec{r}^{2}<\nu^{2} t^{2}$ satisfying the constraint condition of $\mathrm{H}^{4}$. However, it is possible to use other projections.

Let $y^{\prime}(t):=(t, \overrightarrow{0},-\nu t) \ni O$ be antipodal in $S_{t}^{3}$ of the comoving observer $x^{\prime}(t)=$ $(t, \overrightarrow{0}, \nu t)$, with $t \in \mathbb{R}_{\geq 0}$. [17] analysed the locally conformal family of azimuthal projections $f^{\alpha}: \mathcal{T}_{t}\left(S_{t}^{3} \backslash\left\{y^{\prime}\right\}\right) \subset \mathbb{R}_{\eta}^{1,4} \rightarrow \mathbb{R}_{g}^{1,3}$ for the angular coordinate $\gamma \in\left[0, \gamma_{k}\right)$, $\operatorname{mapping}(t, \vec{r}, u) \mapsto\left(f_{\hat{t}}^{\alpha}(t, \vec{r}), f_{\hat{r}}^{\alpha}(t, \vec{r})\right)=(\hat{t}, \overrightarrow{\hat{r}}) \in \mathbb{R}_{g}^{1,3}$ with:

$$
\begin{aligned}
& \overrightarrow{\hat{r}}=f_{\hat{r}}^{\alpha}(t, \vec{r}):=\nu t \frac{\gamma}{\left(1-\gamma / \gamma_{k}\right)^{\alpha}} \vec{k}_{r} \\
& \hat{t}=f_{\hat{t}}^{\alpha}(t, \vec{r})
\end{aligned}
$$

where $f_{\hat{t}}(t, \overrightarrow{0}) \equiv t, \vec{k}_{r}:=\vec{r} / r, r:=|\vec{r}|, \alpha$ is the distortion parameter, and $\gamma_{k}:=\sin ^{-1}\left(\sqrt{k-k^{2} / 4}\right) \in[\pi / 3, \pi)$ is a domain limit according to the parameter $k:=\nu^{-2} \in(0,1]$. Note that it can be also defined the comoving vector $\overrightarrow{\hat{r}}^{\prime}:=\left(t_{\hat{o}} / t\right) \overrightarrow{\hat{r}}$. 
Lagrangian density and symmetries of inhomogeneous hyperconical universes ${ }^{A} T_{E} X 2_{\varepsilon} 8$

The above discussion Eq. (16-19) is equivalent to saying that the observer fixes time coordinate for measurements using a initial time $t_{\hat{o}}$, but it is moving with respect to $O:=(0, \overrightarrow{0}, 0)$. Thus, its reference line is $\widehat{O}:=\mathcal{T}_{t} x^{\prime}\left(t_{\hat{o}}\right)=\left(t_{\hat{o}}, \overrightarrow{0}, \nu t\right) \subset M$ (note that $\widehat{O} \not \subset \mathrm{H}^{4}$ except at $\left.t=t_{\hat{o}}\right)$. This reference line allows us to define the notion of moving charts.

\subsection{Metric tensor}

Let $X \subset \mathrm{H}^{4}$ be any curve. The differential line element $d X$ is easily obtained knowing that $d \vec{r}^{\prime}$ can be decomposed in spherical coordinates as $d \vec{r}^{\prime}=d r^{\prime} \overrightarrow{k_{r}}+r^{\prime} d \Sigma^{\prime} \overrightarrow{k_{\Sigma}}$, where $d \Sigma^{\prime} \overrightarrow{k_{\Sigma}}:=d \theta^{\prime} \overrightarrow{k_{\theta}}+\sin \theta^{\prime} d \phi \overrightarrow{k_{\phi}}$ is orthogonal to radial direction $\overrightarrow{k_{r}}$. According to Eq. (19), and since $d t_{\hat{o}}=0$, the non-zero elements of the metric $g$ for the reference $\widehat{O}$ are:

$$
\begin{aligned}
& g_{00}=2 k^{-1}(b-1)+1 \\
& g_{r r}=-\frac{a^{2}}{b^{2}} \\
& g_{\theta \theta}=-a^{2} r^{\prime 2} \\
& g_{\phi \phi}=-a^{2} r^{\prime 2} \sin ^{2} \theta \\
& g_{0 r}=-\frac{a r^{\prime}}{t_{\hat{o}} b}
\end{aligned}
$$

where $b:=\sqrt{1-k r^{\prime 2} / t_{\hat{o}}^{2}}, \quad k^{-1}:=\nu^{2}=1-\beta_{o}^{2}$ and $a:=t / t_{\hat{o}}$ is the scale factor (see more details in [16]). Note that if $\mathcal{T}_{t_{o}}$ is used instead of $\mathcal{T}_{t}$, it is obtained a static metric equivalent to the FRW universe but without expansion.

Symmetrical spatial elements $g_{i i}$ obtained from Eq. (22) are compatible with some FRW metrics, but elements $g_{00} \neq 1$ and $g_{0 r} \neq 0$, respectively, imply lapse and shift terms, as in the ADM formulation of gravity [19]. To compare $g$ with the FRW metrics, a diagonal version of the metric is given by the coordinate change $t^{\prime}:=t \sqrt{2 k^{-1}(b-1)+1}$, which is equivalent to selecting $g_{00}^{\prime}=1, g_{0 r}^{\prime}=0$ and:

$$
\begin{aligned}
& g_{r r}^{\prime}=g_{r r}-\frac{g_{0 r}^{2}}{g_{00}}=-a\left(t^{\prime}, r^{\prime}\right)^{2} \frac{1-k^{-1}(b-1)^{2}}{b^{2}\left(2 k^{-1}(b-1)+1\right)} \\
& g_{\phi \phi}^{\prime}=-a\left(t^{\prime}, r^{\prime}\right)^{2} r^{\prime 2} \sin ^{2} \theta \\
& g_{\theta \theta}^{\prime}=-a\left(t^{\prime}, r^{\prime}\right)^{2} r^{\prime 2}
\end{aligned}
$$

where $a\left(t^{\prime}, r^{\prime}\right)=t^{\prime} /\left(t_{\hat{o}} \sqrt{2 k^{-1}(b-1)+1}\right)$. Despite this, important differences remain in the FRW metric. Result is an inhomogeneous hypersurface similar to the LemaitreTolman-Bondi (LTB) type [33, 34], but with the same factor scale $a$ for the radial and angular components of the metric (in contrast with the LTB universes, that distinguish between angular and radial expansion). Differences are also found comparing with the McVittie metric, which is an asymptotically spatially flat FLRW metric for largest distances. [35]

To compare with similar FLRW metrics, we can see that the $t^{\prime}$-isochronous hypersurface is similar to a paraboloid, in contrast to the homogeneous hypersphere given by the $t$-isochronous (Fig. 1). 
Metric tensor constructed from $\widehat{O}$ describes a universe with linear expansion, directly proportional to its age or time, $t$. With this, the Hubble parameter is defined as $H:=\dot{a} / a=1 / t$. The most current value of the Hubble parameter is $H_{o}=1 / t_{\hat{o}}$ and, taking the age of the universe as $t_{\hat{o}}=(1.380 \pm 0.004) \cdot 10^{10}$ years $[21,22]$, it is obtained a value $H_{o}=70.9 \pm 0.2 \mathrm{~km} \cdot \mathrm{s}^{-1} \cdot \mathrm{Mpc}^{-1}$ compatible with the local observations $[16,36,11,12]$.

\subsection{Ricci scalar curvature}

Let $R_{\alpha \beta}$ be local coordinates of the Ricci curvature tensor defined for signature $(1,3)$,

$$
R_{\alpha \beta}:=R_{\alpha \mu \beta}^{\mu}=\partial_{\mu} \Gamma_{\alpha \beta}^{\mu}-\partial_{\beta} \Gamma_{\mu \alpha}^{\mu}+\Gamma_{\rho \mu}^{\mu} \Gamma_{\alpha \beta}^{\rho}-\Gamma_{\rho \beta}^{\mu} \Gamma_{\alpha \mu}^{\rho}
$$

where $\Gamma_{\alpha \beta}^{\mu}$ are the Christoffel symbols.

It is found that the manifold is only maximally symmetric in the spatial components $\left(R_{i j}=1 / 3 \cdot R g_{i j}\right)$ at a local scale $\left(r^{\prime}=0\right.$, i.e. $\left.b=1\right)$. In this case, the spatial part of the Ricci tensor $R_{i j}$ approximates to $R_{i j} \approx-2 k(\dot{a} / a)^{2} g_{i j}$. This contrasts with the non-accelerated case for the FRW metric, i.e. $R_{i j}^{F R W}=-2(\dot{a} / a)^{2} g_{i j}^{F R W}-2 K / a^{2} g_{i j}^{F R W}$, where $K$ is the FRW curvature. Taking the Ricci scalar curvature, obtained from the trace $R:=R_{\alpha}^{\alpha}$,

$$
R=-\frac{6 k^{2}}{t^{2}} \frac{2 b-\frac{1}{3}\left(2 b^{2}+1\right)+k-1}{\left(2 b-b^{2}+k-1\right)^{2}}
$$

local limit $\left(r^{\prime}=0\right.$ ) leads to the simplification $R \approx-6 k / t^{2}=-6 /(\nu t)^{2}$, as a 3 -sphere (of radius $\nu t$ ) embedded into $\mathrm{R}^{1,4}$, like in the Anti-de Sitter spaces. For the local universe with $k=1$, the Ricci scalar corresponds to the case of FRW metric with linear scale factor $a=t / t_{\hat{o}}$ and curvature $K=0$, i.e., this is apparently flat under the FRW view.

Despite the assumption of linear expansion with constant factor $\beta_{o}$, this expansion could depend on the spatial coordinates $(\vec{r}, u(\vec{r}))$, of course excluding the time $t$, and then a most general constraint is $|X-O|_{\eta}=\beta(\vec{r}, u) t$. In this case, the resulting curvature $k=1 /\left(1-\beta(\vec{r}, u)^{2}\right)$ also depends on the spatial coordinates. However, we can normalise the local limit as $k(0, u(0))=1$.
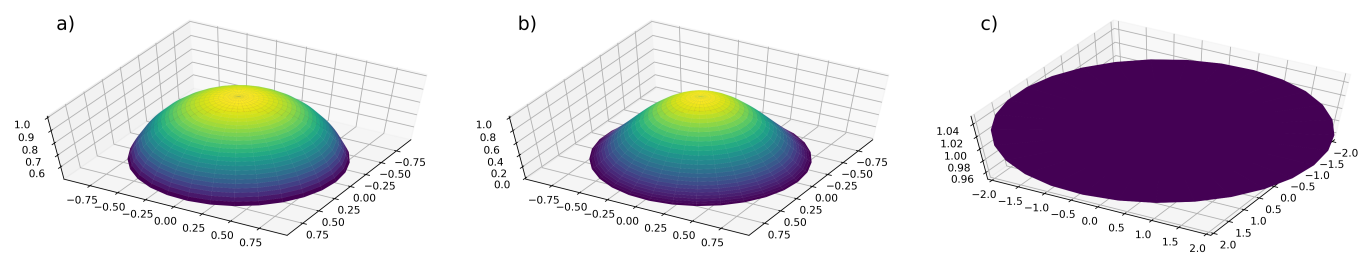

Figure 1. Isochronous hypersurfaces normalised to universe age scale $\left(t_{\hat{o}} \equiv 1\right)$ for: a) Linearly-expanding homogeneous space ( $t$-isochronous), obtained considering coordinates such that $g_{0 r} \neq 0$ and $g_{00} \neq 1$; b) linearly-expanding inhomogeneous space ( $t^{\prime}$-isochronous), obtained considering coordinates such that $g_{0 r}^{\prime}=0$ and $g_{00}^{\prime}=1$; c) apparently-accelerated flat FRW universe according to locally conformal map (distorted azimuthal projection $\alpha=0.28$, Eq. (20)) 
Lagrangian density and symmetries of inhomogeneous hyperconical universes ${ }^{A} T_{E} X{ }_{2} 10$

\section{Results I: Derivation of modified Lagrangian density}

\subsection{Local Friedmann equations}

According to the new theoretical frame, a modified Lagrangian density can be obtained from the local Friedmann equations, assuming GR valid for that scale. While most of studies find the Universe metric as a solution of the Friedmann equations, the work of [16] found new Friedmann equations and the value of the energy parameters as a solution of the proposed inhomogeneous "void" metric. Locally, equations with positive curvature $\left(k_{o}=1\right)$ is compatible with the flat FRW case (assuming linear expansion). Additionally, taking $\rho_{\text {crit }}(t)=\rho_{o}+\rho_{\Lambda}$, where $\rho_{\text {crit }}(t):=3 k_{o} H^{2} / 8 \pi G$ and $\rho_{\Lambda}:=\Lambda / 8 \pi G$, the hyperconical model leads to:

$$
\begin{aligned}
& 4 \pi \mathrm{G} \rho_{o}(1+w)=\frac{k_{o}}{t^{2}} \\
& \Lambda t^{2}=k_{o} \frac{1+3 w}{1+w}
\end{aligned}
$$

where $w$ is the parameter of the matter state equation. If $\Lambda$ is finite and constant, it is obtained that $\Lambda=0, \mathrm{G}$ is constant, and $w=-1 / 3$ for any time $t$ [16]. In addition, if mass is homogeneously and isotropically distributed throughout the Universe, we can define a mass function $m(r):=4 \pi \rho_{o} r^{3} / 3$, and Eq. 32 can be formulated as:

$$
\frac{k_{o}}{t^{2}}=\frac{8 \pi \mathrm{G} \rho_{o}}{3}=\frac{2 \mathrm{G} m(r)}{r^{3}}
$$

Note that for $k_{o}=1$, we get $\dot{r}=r / t=\sqrt{2 \mathrm{Gm}(r) / r}$, i.e. Hubble's law is an escape velocity. If this approach is considered for the spatial components of the hyperconical metric, $g_{r r}=-a(t)^{2} /\left(1-r^{2} / t^{2}\right)$, it locally approaches to the Schwarzschild components, $g_{r r}=-a(t)^{2} /(1-2 \mathrm{Gm}(r) / r)$, as expected for inhomogeneous metrics (e.g. LTB and McVittie metrics [33, 35]).

Summarising, inhomogeneous metric with a locally valid GR leads to $\Omega_{\Lambda}=$ $\rho_{\Lambda} / \rho_{\text {crit }}=0$, but if that metric is projected on a flat FRW metric (forcing GR to be generally valid), the inhomogeneity is absorbed as an acceleration and dark energy is $\Omega_{\Lambda}=0.6937181(2)([[17]])$.

\subsection{Modified Lagrangian density}

From the assumption that GR is valid at local scale, it follows that the expansion of the Universe is compatible with the equation of state $w=-1 / 3$ for the energy density, i.e., $\rho_{o}=\rho_{\text {crit }}$ and $p_{o}=-\rho_{\text {crit }} / 3[16]$. This simplyfies the Einstein field equations to:

$$
G_{\alpha \beta}=8 \pi \mathrm{G} T_{\alpha \beta}=8 \pi \mathrm{G}\left(T_{\alpha \beta}^{o}+T_{\alpha \beta}^{m}\right),
$$

where $T^{m}{ }_{\alpha \beta}$ is the stress-energy tensor of the ordinary matter-energy and $T_{\alpha \beta}^{o}=$ $\left(\rho_{o}+p_{o}\right) u_{\alpha} u_{\beta}-p_{o} g_{\alpha \beta}$ is the stress-energy tensor of the background space-time, with $w=-1 / 3$ and energy density equal to $\rho_{\text {crit }}=3 k_{o} / 8 \pi \mathrm{G} t^{2}$. We deduce the total 
Lagrangian density as locally equal to:

$$
\mathcal{L} \approx \frac{1}{16 \pi \mathrm{G}}\left(R+\frac{6 k_{o}}{t^{2}}\right)+\mathcal{L}_{\mathcal{M}}
$$

where $\mathcal{L}_{\mathcal{M}}$ is the Lagrangian term of the ordinary mass-energy. Note that the curvature term $R+6 k_{o} / t^{2}$ corresponds to the local difference between the total Ricci curvature $R$ and the local limit of the Ricci curvature estimated for the empty hyperconical universe, $R_{u} \approx-6 k_{o} / t^{2}$. Therefore, a modified gravity is required for the general case. The simplest modification for the Lagrangian density is given by the general difference $\Delta R:=R-R_{u}$, that is

$$
\mathcal{L}=\frac{\Delta R}{16 \pi \mathrm{G}}+\mathcal{L}_{\mathcal{M}}
$$

which corresponds to a simple type of modified gravity Lagrangian density, which leads to equations similar than those obtained using a flat FRW universe.

The modification of the Lagrangian can be interpreted as a redefinition of the energy density baseline or "vacuum energy", given by the global curvature $R_{u}$, i.e. the critical energy density.

\section{Results II: Analysis of Symmetries}

\subsection{Euler-Lagrange equations}

Let $\mathcal{S}$ be the action functional for describing the hyperconical manifold $\mathrm{H}^{4}$ embedded in $M$, it corresponds to a constant $m \neq 0$ multiplied by the distance $\tau:=|X-O|_{\eta}$ of the relative path of $X-O \in M$ under the metric $\eta$, and the Lagrangian $L$ coincides with the constant product $m \beta_{o}$. That is,

$$
\mathcal{S}[\tau(t)]=\int_{\mathrm{H}^{4}} m d \tau=\int_{0}^{t} m \beta_{o} d t
$$

This retrieves the constraint $|X-O|_{\eta}=\beta_{o} t$, viewed under the chart $C$. However, if a path $x=x(t) \subset \mathbb{R}_{g}^{1,3}$ is analysed respect to the chart $D$, the new action is given by $\tau(t)=|x(t)|_{g}:$

$$
\mathcal{S}[X(t)]=\int_{0}^{t} m \sqrt{g_{\alpha \beta} \frac{d x^{\alpha}}{d \lambda} \frac{d x^{\beta}}{d \lambda}} d \lambda \cong \int_{0}^{t} \frac{m}{2} g_{\alpha \beta} \dot{x}^{\alpha} \dot{x}^{\beta} d \tau
$$

where $\dot{x}^{\mu}:=d x^{\mu} / d \tau$, and it is used the homomorphism between the extremal $\delta \sqrt{\bullet}=0$ and $\frac{1}{2} \delta \bullet=0$. Therefore, the Lagrangian functional for $\mathbb{R}_{g}^{1,3}$ can be taken as $L=$ $\frac{1}{2} g_{\alpha \beta} \dot{x}^{\alpha} \dot{x}^{\beta}$. Euler-Lagrange equations provided by the above action are the corresponding geodesic equations $\ddot{x}^{\rho}=-\Gamma_{\alpha \beta}^{\rho} \dot{x}^{\alpha} \dot{x}^{\beta}$, being $\Gamma_{\alpha \beta}^{\rho}$ the Christoffel symbols of second kind, 
Lagrangian density and symmetries of inhomogeneous hyperconical universes ${ }^{A} T_{E} X 2_{\varepsilon} 12$ the non-vanishing ones being:

$$
\begin{array}{ll}
\Gamma_{j 0}^{i} & =\frac{\dot{a}}{a} \delta_{j}^{i} \\
\Gamma_{i j}^{0} & =-g_{i j} \frac{\dot{a}}{a} \frac{k(1-b)}{2 b-b^{2}+k-1} \\
\Gamma_{r r}^{r} & =\frac{1}{b^{2}} \frac{k r^{\prime}}{t_{\hat{o}}^{2}} \frac{b+k-1}{2 b-b^{2}+k-1} \\
\Gamma_{\phi \phi}^{r} & =-r^{\prime} \sin ^{2} \theta \frac{b+(k-2) b^{2}+b^{3}}{2 b-b^{2}+k-1} \\
\Gamma_{\theta \theta}^{r} & =-r^{\prime} \frac{b+(k-2) b^{2}+b^{3}}{2 b-b^{2}+k-1} \\
\Gamma_{\theta r}^{\theta}=\Gamma_{\phi r}^{\phi} & =\frac{1}{r^{\prime}} \\
\Gamma_{\phi \phi}^{\theta} & =-\sin \theta \cos \theta \\
\Gamma_{\phi \theta}^{\phi} & =\cot \theta
\end{array}
$$

Angular velocities $\dot{\theta}$ and $\dot{\phi}$ remain spherical symmetry as in the flat Minkowski universe. However, time and radial coordinates present a flux:

$$
\begin{aligned}
& \ddot{t}=-\frac{k(1-b)}{2 b-b^{2}+k-1} \frac{t}{t_{\hat{o}}^{2}}\left(\frac{\dot{r}^{\prime 2}}{b^{2}}+r^{\prime 2} \dot{\bullet}^{\prime 2}\right) \\
& \ddot{r}^{\prime}=-2 \frac{\dot{r}^{\prime} t}{t}-\frac{\frac{k r^{\prime}}{b^{2} t_{\hat{o}}^{2}}(b+k-1)}{2 b-b^{2}+k-1} \dot{r}^{\prime 2}+\frac{b+(k-2) b^{2}+b^{3}}{2 b-b^{2}+k-1} r^{\prime} \dot{\Sigma}^{2}
\end{aligned}
$$

where $\dot{\vec{r}}^{2}:=-g_{i i} \dot{x}^{i} \dot{x}^{i}$ is the square of the spatial velocity, and it is taken $H:=\dot{a} / a=1 / t$. The local limit $\left(r^{\prime} / t_{\hat{o}}<<1\right)$ without rotation $(\dot{\Sigma}=0)$ for Eq. (48) is only non-zero at the third order, and Eq. (49) is non-zero for the first order:

$$
\begin{aligned}
& \ddot{t} \approx-\frac{k r^{\prime 2} t}{2 t_{\hat{o}}^{4}} \dot{r}^{\prime 2} \\
& \ddot{r}^{\prime} \approx-\frac{2}{t} \dot{r}^{\prime} \dot{t}-\frac{k r^{\prime}}{t_{\hat{o}}^{2}} \dot{r}^{\prime 2}
\end{aligned}
$$

where $\dot{t}=d t / d \tau:=\gamma$, and therefore $\ddot{t}=\gamma \dot{\gamma}$, while $\dot{r}^{\prime}=\gamma \dot{r}^{\prime}$ and $\ddot{r}^{\prime}=\gamma^{2}\left(\ddot{r}^{\prime}+\dot{\gamma} \dot{r}^{\prime}\right)$. Considering $\ddot{r}^{\prime} \approx \gamma^{2} \ddot{r}^{\prime}$, and taking the comoving relation $r^{\prime}:=\left(t_{\hat{o}} / t\right) r$, Eq. (50) and (51) are approximately:

$$
\begin{aligned}
& \dot{\gamma} \approx-\gamma \frac{k r^{2}}{2 t^{3}}\left(\dot{r}-\frac{r}{t}\right)^{2} \\
& \ddot{r} \approx-\frac{k r}{t^{2}}\left(\dot{r}-\frac{r}{t}\right)^{2}
\end{aligned}
$$

As might be expected, the comoving case $(\dot{r}=r / t)$ is a solution of the Eq. (53), for which the linear expansion is stationary $(\ddot{r}=0=\dot{\gamma})$. 


\subsection{Modified Gravity and ADM equations}

Applying the Einstein field equations to the studied metric, it is obtained that energy density of the local universe is equal to $R_{u} \approx-6 k_{o} / t^{2}$ in appropriate units (see 3). According to this, total Lagrangian density of our universe is given by the following modified gravity action:

$$
\mathcal{S}=\int d^{4} x \mathcal{L}=\int d^{4} x \sqrt{-g}\left(R-R_{u}+\mathcal{L}_{M}\right)
$$

where $\mathcal{L}_{M}$ is the term of mass.

As space-time is foliated into a family of space-like surfaces $\Sigma_{t}$, the spatial submanifold can be interpreted as an embedding into the space-time (in our case $\mathrm{H}^{4}$ ). And therefore, the ADM formalism can be used to obtain a set of equations of motion for the generalised coordinates, which corresponds to the spatial elements $g_{i j}$ of the metric (see Sec. 1.3). We consider the local limit

$$
R_{u} \approx-\frac{6 k_{o}}{t^{2}}=-\frac{k_{o}}{2}\left(g^{i j} \partial_{t} g_{i j}\right)^{2}
$$

and then Lagrangian density can be rewitten according to ADM formalism (Eq. (9)), but with an additional term from the curvature $R_{u}$ :

$$
\mathcal{L}=-g_{i j} \partial_{t} \pi^{i j}+l h+2 l_{i} \pi_{; j}^{i j}-2 \partial_{i} s^{i}+\sqrt{-g} R_{u}
$$

where $l:=\left(g^{00}\right)^{-1 / 2}$ is the lapse, $l_{i}:=g_{0 i}$ is the shift, $h$ is the Hamiltonian constraint (Eq. (11)), $s^{i}$ is an auxiliary momenta (Eq. (12)), but $\pi^{i j}$ is now the modified conjugate momenta (which slightly different to Eq. (10)):

$$
\pi^{i j}=\sqrt{-g}\left[\left(\Gamma_{p q}^{0}-g_{p q} \Gamma_{r s}^{0} g^{r s}\right) g^{i p} g^{j q}-\frac{\partial R_{u}}{\partial\left(\partial_{t} g_{i j}\right)}\right]
$$

where $g:=\operatorname{det} g_{\mu \nu}$ is the determinant of the metric, in contrast with $g_{s}:=\operatorname{det} g_{i j}$, that is the determinant of the spatial metric.

For the studied metric, Eq. (41) is locally as $\Gamma_{i j}^{0} \approx-g_{i j}\left(k_{o} r^{2}\right) /\left(2 t_{\hat{o}}^{2} t\right)$ and therefore Eq. (57) is simplified as:

$$
\begin{aligned}
& \pi^{i i} \approx \sqrt{-g}\left[\left(-g_{i i} \frac{k_{o} r^{\prime 2}}{2 t_{\hat{o}}^{2} t}+g_{i i} \delta_{m}^{m} \frac{k_{o} r^{\prime 2}}{2 t_{\hat{o}}^{2} t}\right)\left(g^{i i}\right)^{2}+k_{o}\left(g^{i i}\right)^{2} \partial_{t} g_{i i}\right] \approx \\
& \approx \sqrt{-g} \frac{k_{o} g^{i i}}{t}\left(\frac{k_{o} r^{\prime 2}}{t_{\hat{o}}^{2}}+2\right) \approx 2 \sqrt{-g} \frac{k_{o} g^{i i}}{t}=\sqrt{-g} k_{o}\left(g^{i i}\right)^{2} \partial_{t} g_{i i}
\end{aligned}
$$

where the metric determinant is locally $g \approx g_{s} \approx-a(t)^{6} r^{4} \sin ^{2} \theta$, since $g^{00} \approx 1$. The $\mathrm{ADM}$ equations for the metric evolution are given by the Eq. (13), and using that $\pi_{i j}=g_{i a} g_{j b} \pi^{a b}$ and $g_{i j}=0$ for $j \neq i>0$, it is obtained that $\pi_{i i}=\pi g_{i i}=\pi^{i i} g_{i i}{ }^{2}$. That is:

$$
\partial_{t} g_{i i}=\frac{1}{\sqrt{-g_{s} g^{00}}} \pi^{i i} g_{i i}^{2}+2 \nabla_{i} g_{0 i}
$$

Trivially, it is obtained that:

$$
k_{o}=1
$$


where it is used that the covariant derivative of the shift $g_{0 r}$ is locally zero:

$$
\nabla_{r} g_{0 r}=\partial_{r} g_{0 r}-\Gamma_{0 r}^{r} g_{r r}-\Gamma_{r r}^{r} g_{0 r} \approx-\frac{a}{t_{\hat{o}}}+\frac{a^{2}}{t}=0
$$

Therefore, the evolution of the used metric is compatible with the ADM formalism applied to the modified Lagrangian density $\mathcal{L}$.

\subsection{Killing Vectors}

As is well known, the Killing vectors $K^{\mu}$ correspond to the infinitesimal generators of the isometries of the metric tensor [37, 38], hence, in terms of the Levi-Civita connection, we have the identity $g\left(\nabla_{X} K, Y\right)=g\left(X, \nabla_{Y} K\right)$. In local coordinates the Killing equation is given by

$$
\nabla_{\mu} K_{\nu}+\nabla_{\nu} K_{\mu}=0
$$

An important property of the Killing vectors is the conservation of a quantity $Q$ respect to the affine parameter $\lambda$, i.e. $d Q / d \lambda=0$, where:

$$
Q:=K_{\mu} \frac{d x^{\mu}}{d \lambda}
$$

Note that this quantity corresponds to the Noether charge conserved by the coordinate transformation

$$
x^{\mu} \longrightarrow x^{\mu}+d \varphi^{\mu}(\lambda)=x^{\mu}+K^{\mu} d \lambda
$$

and one can identify $Q=K^{\mu} \pi_{\mu}$ where $\pi_{\mu}:=\partial L / \partial \dot{x}^{\mu}$ is the generalised momentum and $L$ is the Lagrangian functional in $\mathrm{R}_{g}^{1,3}$.

For our case, the first Killing vector is determined by the independence of the metric Eq. (22) with respect to the coordinate $\phi$, hence $K_{(1)}=\delta_{\phi}^{\mu} \partial_{\mu}=(0,0,0,1)$. The other two Killing vectors, resulting from the angular symmetry, can be obtained from Eq. (62) as $K_{(2)}=(0,0,-\cos \phi, \cot \theta \sin \phi)$ and $K_{(3)}=(0,0, \sin \phi, \cot \theta \cos \phi)$, respectively. These vector fields are easily seen to generate a copy of $\mathfrak{s o}(3)$ the angular momenta $\ell_{(i)}=g_{\mu \nu} K_{(i)}^{\mu} d x^{\nu} / d \lambda$ are preserved, according to Eq. (63). For simplicity, we choose the coordinates with $\theta=\pi / 2$, so that the direction of angular momentum is on the $z$-axis (i.e. $\theta=0$ ) and thus the motion is in the $x y$-plane:

$$
\ell_{(1)}=a(t)^{2} r^{\prime 2} \frac{d \phi}{d \lambda}=r^{2} \frac{d \phi}{d \lambda}
$$

where for computational convenience we use that $r=a(t) r^{\prime}$ and $\lambda=\tau$.

Concerning the translation symmetries, we have to distinguish between local and global symmetries. Spatial translations are clearly not global for the analysed metric, but a temporal translation is found for the static conformal metric $g^{\prime}$ given by $d \lambda^{2}:=$ $a(t)^{-2} d X^{2}$, i.e. changing the time coordinate as $d \eta:=a(t)^{-1} d t$. The time-like conformal Killing vector is thus $K_{(\eta)}=\partial_{\eta}$. This implies the conservation of the energy

$$
E\left(r^{\prime}\right)=g_{\mu \nu}^{\prime} K_{(\eta)}^{\nu} \frac{d x^{\mu}}{d \lambda}=\left(2 k^{-1}\left(b\left(r^{\prime}\right)-1\right)+1\right) \frac{d \eta}{d \lambda}
$$


in a static universe. Note that, locally,

$$
E\left(r^{\prime}\right) \approx\left(1+2 \Phi\left(r^{\prime}\right)\right) \gamma
$$

where $2 \Phi\left(r^{\prime}\right):=-r^{\prime 2} / t_{\hat{o}}^{2}$ and $\gamma=d t / d \tau=d \eta / d \lambda$. If we take into account the relation between $r^{\prime 2} / t_{\hat{o}}^{2}$ and the density of the Universe (see Eq. (34) in the Appendix), then Eq. (67) can be interpreted as an analogue to the potential gravitational energy. Spatial translations close to $r^{\prime} \approx 0$ are local symmetries for the three orthogonal spatial directions $x^{i \prime}$, with $i=1,2,3$, where $x^{1 \prime}:=r^{\prime} \sin \theta \cos \phi, x^{2 \prime}:=r^{\prime} \sin \theta \sin \phi$,

$x^{3 \prime}:=r^{\prime} \cos \theta$. As the metric is locally flat for these coordinates, the space-like Killing vectors are trivially $K_{\left(i^{\prime}\right)}=\partial_{i^{\prime}}$, and the linear momentum conserved is $P^{i}=a(t) d x^{i \prime} / d \tau$. Finally, the three Lorentz boosts $B_{(i)}=x^{i} \partial_{0}-t \partial_{i}$ are symmetries for the ordinary coordinates $x^{i}=a(t) x^{i \prime}$. Summarising, there are $10=3+1+3+3$ Killing vectors of either global/local type, corresponding to the three global angular rotations $K_{(i)}$, the temporal translation $K_{(\eta)}$ for the conformal static Universe, as well as three local spatial translations $K_{\left(i^{\prime}\right)}$ and three local boosts $B_{(i)}$ as it is locally found in GR [37].

\section{Conclusions}

This work explores some of the symmetry properties of inhomogeneous hyperconical universes. The metric tensor is obtained assuming that the Universe expands linearly and independently of its matter content. In particular, we assume that any observer is placed on either a 3-spheroid or 3-hyperboloid with radial expansion (4-hypercone). The key assumption is that the proper time of each observer is preserved, and thus fixes an initial reference time, even though its position varies in time due to the expansion.

The Lagrangian analysis for the proposed model is in agreement with the observations. The comoving trajectories are solutions of the Euler-Lagrange equations, corresponding to the trivial case with stationary linear expansion. However, for the ADM formalism, we require a modified Gravity Lagrangian density in order to ensure that the evolution of the metric is consistent at local scale, obtaining positive curvature of $(k=1)$. This is also consistent with the best prediction of dark energy density, also found for $k=1$.

With respect to the Killing vectors, it is shown that the angular rotations are global symmetries, while spatial translations are merely local symmetries. The corresponding preserved linear momentum presents a scaling factor $a(t)$, as expected. Finally, the three Lorentz boosts are also local symmetries (for the ordinary orthogonal frame).

For the most of the analysed cases, the symmetric properties are referring to the local case and assuming that the parameter $k(\vec{r}, u(\vec{r}))=k_{o}$ is globally homogeneous (leading to a radial inhomogeneous metric). That is, the proposed model should be understood as an alternative model to be analysed in greater depth, adding corrections from higher order terms or other approximations. We observe that the gauge Noether symmetries are potentially relevant in the analysis of the field equations in the linearly expanded system. Whether the related conservation currents derived from this approach 
provide an additional insight into the interpretation of the Hubble parameter compatible with observational data is still an open question. For instance, we can interpret the radial inhomogeneity of the metric as an apparent radial acceleration, which breaks all the non-rotational local symmetries at large distances.

\section{Acknowledgments}

During the preparation of this work, RCS was financially supported by the research project MTM2016-79422-P of the AEI/FEDER (EU).

\section{References}

[1] Spergel D N et al (WMAP collaboration) 2007. Wilkinson Microwave Anisotropy Probe (WMAP) Three Year Results: Implications for Cosmology. Astrophys. J. Suppl. 170 377-408.

[2] Brax P 2013. Screening mechanisms in modified gravity. Class. Quantum Grav. 30, 214005. doi:10.1088/0264-9381/30/21/214005

[3] Herfray Y, Krasnov K, Shtanov Y 2016. Anisotropic singularities in chiral modified gravity. Class. Quantum Grav. 33 235001. doi:10.1088/0264-9381/33/23/235001

[4] Cai Y-F, Capozziello S, De Laurentis M, Saridakis E N 2016. f(T) teleparallel gravity and cosmology Rept. Prog. Phys. 79, 106901. DOI: 10.1088/0034-4885/79/10/106901

[5] Krššák M, van den Hoogen R J, Pereira J G, Böhmer C G and Coley A A. 2019. Teleparallel theories of gravity: illuminating a fully invariant approach.Class. Quantum Grav. 36183001

[6] Wiltshire and David L 2007. Exact Solution to the Averaging Problem in Cosmology. Phys. Rev. Let. 99 251101. doi:10.1103/physrevlett.99.251101.

[7] Preston A W H and Morris T R 2014 Cosmological back-reaction in modified gravity and its implications for dark energy J. Cosmol. Astropart. Phys., 2014 017-017 doi:10.1088/1475$7516 / 2014 / 09 / 017$

[8] Dam L H, Heinesen A and Wiltshire D L 2017. Apparent cosmic acceleration from Type Ia supernovae. Mon. Not. R. Astron Soc. 472 835-851. doi:10.1093/mnras/stx1858

[9] Speck J 2018. The Maximal Development of Near-FLRW Data for the Einstein-Scalar Field System with Spatial Topology $\mathbb{S}^{3}$. Commun. Math. Phys. 364 879-979.

[10] Lima J A S 2007. Age of the Universe, Average Deceleration Parameter and Possible Implications for the End of Cosmology [arXiv:0708.3414 [astro-ph]].

[11] Riess A G, Casertano S, Yuan W, Macri L M and Scolnic D 2019 Large Magellanic Cloud Cepheid Standards Provide a 1\% Foundation for the Determination of the Hubble Constant and Stronger Evidence for Physics beyond $\Lambda$ CDM The Astrophysical Journal 87685.

[12] Knox L and Millea M 2020 The Hubble Hunter's Guide Phys. Rev. D 101043533 arXiv:1908.03663. DOI: 10.1103/PhysRevD.101.043533

[13] Melia F and Abdelqader M 2009. The cosmological spacetime Int. J. Modern Phys. D 181889

[14] Benoit-Lévy A and Chardin G 2012 Introducing the Dirac-Milne Universe Astron. Astrophys. 537 id.A78

[15] Melia F and Shevchuk A S H 2012 The Rh = ct Universe, Mon. Not. R. Astron Soc. 4192579

[16] Monjo R 2017 Study of the observational compatibility of an inhomogeneous cosmology with linear expansion according to SNe Ia Phys. Rev. D 96103505

[17] Monjo R 2018 Geometric interpretation of the dark energy from projected hyperconical universes. Phys. Rev. D 98043508

[18] Arnowitt R, Deser S and Misner C 2008 Republication of: The dynamics of General Relativity. Gen. Relelativ. Gravit. 40 (2008) 1997-2027 
[19] Deruelle N, Sasaki M, Sendouda Y and Yamauchi D 2010 Hamiltonian formulation of $\mathrm{f}$ (Riemann) theories of Gravity. Prog. Theor. Phys. 123 169-185

[20] McFeron D and Székelyhidi G 2012 On the Positive Mass Theorem for Manifolds with Corners Commun. Math. Phys. 313 425-443

[21] Planck Collaboration 2016 Planck 2015 results. XIII. Cosmological parameters Astron. Astrophys. 596 A13

[22] Planck Collaboration 2018 Planck 2018 results. VII. Cosmological parameters [arXiv:1807.06209 [astro-ph.CO]] (2018)

[23] Buchert T, Larena J and Alimi J-M 2006 Correspondence between kinematical backreaction and scalar field cosmologies - the "morphon field" Class. Quantum Grav. 23 6379-6408. doi:10.1088/0264-9381/23/22/018

[24] Nojiri S and Odintsov S D 2007, Introduction to modified gravity and gravitational alternative for dark energy Int. J. Geom. Methods Mod. Phys. 04 115-145

[25] Ellis G F R. 2011. Inhomogeneity effects in cosmology Class. Quantum Grav., 28164001

[26] Olmo G J 2011 Palatini approach to modified Gravity: $\mathrm{f}(\mathrm{R})$ Theories and beyond Int. J. Modern Phys. D 20 (2011) 413-462

[27] Buchert T, Mourier P and Roy X 2020 On average properties of inhomogeneous fluids in general relativity III: general fluid cosmologies Gen. Relativ. Gravit., 52 doi:10.1007/s10714-020-02670-6.

[28] Peskin M E and Schroeder D V 2018, An Introduction to Quantum Field Theory (CRC Press, New York.)

[29] Achour J B, Crisostomi M, Koyama K, Langlois D, Noui K and Tasinato G 2017 Degenerate higher order scalar-tensor theories beyond Horndeski up to cubic order J. High Energy Phys. 2016100.

[30] Wise D K 2015 Holographic Special Relativity Adv. Theor. Math. Phys. 19 1017-1041

[31] Gryb S 2015 Observing shape in spacetime Gen. Relativ. Gravit. 4737.

[32] Eardley D, Isenberg J, Marsden J et al. 1986 Homothetic and conformal symmetries of solutions to Einstein's equations Commun. Math. Phys. 106 137-158.

[33] Yan X-P, Liu D-. and Wei H, Age Problem in Lemaître-Tolman-Bondi Void models 2015 Age Problem in Lemaître-Tolman-Bondi Void models Phys. Lett. B $\mathbf{7 4 2}$ 149-159.

[34] Yoo J, Watanabe Y 2012 Theoretical Models of Dark Energy Int. J. Modern Phys. D 211230002. doi:10.1142/s021827181230002

[35] Pérez D, Romero G E, Combi L and Gutiérrez E. 2019. A note on geodesics in inhomogeneous expanding spacetimes. Class. Quantum Grav. 36055002.

[36] Lin M-X, Benevento G, Hu W, Raveri 2019 Acoustic Dark Energy: Potential Conversion of the Hubble Tension Phys. Rev. D 100 6, 063542 doi: 10.1103/PhysRevD.100.063542

[37] Chen P, Wang M and Yau S. 2015. Conserved Quantities in General Relativity: From the QuasiLocal Level to Spatial Infinity. Commun. Math. Phys. 338 31-80.

[38] Paliathanasis A. 2016. f(R)-gravity from Killing tensors. Class. Quantum Grav. 33075012. doi:10.1088/0264-9381/33/7/075012 